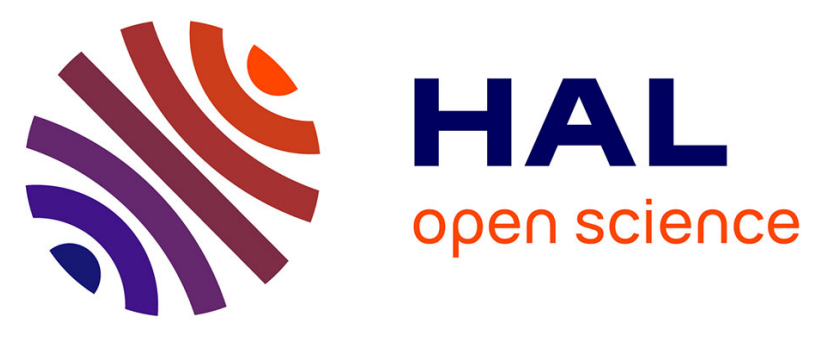

\title{
Incidence of bloodstream infections and predictive value of qualitative and quantitative skin cultures of patients with overlap syndrome or toxic epidermal necrolysis: A retrospective observational cohort study of 98 cases
} Aude Lecadet, Paul-Louis Woerther, Camille Hua, Audrey Colin, Camille Gomart, Jean-Winoc Decousser, Armand Mekontso Dessap, Pierre Wolkenstein, Olivier Chosidow, Nicolas de Prost, et al.

\section{- To cite this version:}

Aude Lecadet, Paul-Louis Woerther, Camille Hua, Audrey Colin, Camille Gomart, et al.. Incidence of bloodstream infections and predictive value of qualitative and quantitative skin cultures of patients with overlap syndrome or toxic epidermal necrolysis: A retrospective observational cohort study of 98 cases. Journal of The American Academy of Dermatology, 2019, 81, pp.342 - 347. 10.1016/j.jaad.2019.03.030 . hal-03488144

\author{
HAL Id: hal-03488144 \\ https://hal.science/hal-03488144
}

Submitted on 20 Dec 2021

HAL is a multi-disciplinary open access archive for the deposit and dissemination of scientific research documents, whether they are published or not. The documents may come from teaching and research institutions in France or abroad, or from public or private research centers.
L'archive ouverte pluridisciplinaire HAL, est destinée au dépôt et à la diffusion de documents scientifiques de niveau recherche, publiés ou non, émanant des établissements d'enseignement et de recherche français ou étrangers, des laboratoires publics ou privés.

\section{(ㄷ)(1) $\$$}

Distributed under a Creative Commons Attribution - NonCommerciall 4.0 International 


\section{Incidence of bloodstream infections and predictive value of qualitative and quantitative} skin cultures of patients with overlap syndrome or toxic epidermal necrolysis: a retrospective observational cohort study of 98 cases.

A. Lecadet $^{1}$, M.D., PL. Woerther ${ }^{2,5}$, M.D., PhD., C. Hua ${ }^{1,4,5}$, M.D., A. Colin ${ }^{1,4}$, C. Gomart $^{2}$, Pharm.D., MSc, JW. Decousser ${ }^{2,5}$, Pharm.D., Ph.D., A. Mekontso Dessap ${ }^{3,4,}$ ${ }^{5}$, M.D., PhD., P. Wolkenstein ${ }^{1,4,5}$, M.D., Ph.D., O. Chosidow ${ }^{1,4,5}$, M.D., Ph.D, N. de Prost $^{3,4,5 *}$, M.D., Ph.D., and S. Ingen-Housz-Oro ${ }^{1,4,6 *}$, M.D.

(1)

\section{Affiliations :}

1. Department of Dermatology, AP-HP Hôpital Henri Mondor, 51 avenue du Maréchal de Lattre de Tassigny, 94010 Créteil, France, Université Paris Est UPEC, Créteil France

2. Department of Microbiology, AP-HP Hôpital Henri Mondor, 51 avenue du Maréchal de Lattre de Tassigny, 94010 Créteil, France, Université Paris Est UPEC, Créteil France

3. Medical Intensive Care Unit, AP-HP Hôpital Henri Mondor, 51 avenue du Maréchal de Lattre de Tassigny, 94010 Créteil, France, Université Paris Est UPEC, Créteil France

4. National Reference Center for Toxic Bullous Dermatosis, AP-HP Hôpital Henri Mondor, 51 avenue du Maréchal de Lattre de Tassigny, 94010 Créteil, France, Université Paris Est UPEC, Créteil France

5. Université Paris-Est Créteil Val de Marne, UPEC, Créteil, France

6. EA7379, UPEC, Créteil, France

* Contributed equally 
23

24

\section{Corresponding author:}

Dr. Saskia Ingen-Housz-Oro, MD

Department of Dermatology, AP-HP Hôpital Henri Mondor, 51 avenue du Maréchal de Lattre de Tassigny

94010 Créteil, France

Tel: +33149812536

Fax: +33149812508

Email : saskia.oro@aphp.fr

\section{Funding sources: None}

IRB approval status: Approved

Conflicts of Interest Disclosure: The authors have no conflicts of interest to declare

Prior presentation: Oral communication in the Journées Dermatologiques de Paris, Paris, France, December 2018

Reprint requests: Not available.

Abstract word count: 198

Capsule summary word count: 49

Text word count: 2014

References: 14 
43

44

45

46

47

48

49

50

51

52

53

54

55

56

57

58

59

60

61

62

63

\section{5} 6 7 8 59 60 61

Table : 1

Figures : 2

Key words: epidermal necrolysis, Lyell syndrome, SJS-TEN overlap syndrome, skin culture, bloodstream infection, blood culture

\section{Capsule summary}

- Bloodstream infections are a frequent complication of epidermal necrolysis.

- Out of 98 patients, $46(46.9 \%)$ had bloodstream infections [S. aureus, $\mathrm{n}=17(36.9 \%)$ and $P$. aeruginosa, $\mathrm{n}=17(36.9 \%)]$. Negative predictive values of skin cultures were $89.4 \%$ and $80.9 \%$, respectively.

- Skin cultures may help predicting the pathogens involved in bloodstream infections. 62 63 
64

65

66

67

68

69

\section{Abstract}

Background: Epidermal necrolysis (EN) involving $\geq 10 \%$ body surface area (BSA) is often complicated by bacterial infections.

Objective: To describe the epidemiology of bloodstream infections (BSIs) in EN involving a $\mathrm{BSA} \geq 10 \%$ and the diagnostic performances of skin cultures for predicting the pathogen(s) isolated from BSIs.

Methods: This retrospective single-center observational study was conducted between 2009 and 2017. All patients referred at the acute phase for $\mathrm{EN}$ involving a $\mathrm{BSA} \geq 10 \%$ were included. All clinical and bacteriological relevant data were collected (blood and skin cultures results, number, severity and time of BSI). Sensitivity, specificity, and predictive values of skin cultures and impact of the bacterial inoculum were investigated.

Results: Out of 98 patients, $46(46.9 \%)$ had $\geq 1$ BSI episode during the hospital stay [Staphylococcus aureus $(\mathrm{n}=17,36.9 \%)$ and Pseudomonas aeruginosa $(\mathrm{n}=17,36.9 \%)]$. Skin cultures were concordant with blood cultures in 32 cases $(71.1 \%)$. The positive and negative predictive values were $57.7 \%$ and $89.4 \%$ for $S$. aureus and $50.0 \%$ and $80.9 \%$ for $P$. aeruginosa, respectively. BSI increased with cutaneous inoculum of S. aureus.

Limitations: Retrospective monocentric design and few total number of BSI.

Conclusion: Skin cultures for $S$. aureus and $P$. aeruginosa may help in predicting the pathogens involved in bloodstream infections. 
86

87

88

89

90

91

92

93

94

95

96

97

98

99

100

101

102

103

104

Abbreviations and acronyms list:

$\mathrm{BSI}=$ bloodstream infection

$\mathrm{BSA}=$ body surface area

$\mathrm{CFU}=$ colony forming unit

$\mathrm{EN}=$ epidermal necrolysis

$\mathrm{NPV}=$ negative predictive value

$\mathrm{OR}=$ odds ratio

P. aeruginosa $=$ Pseudomonas aeruginosa

$\mathrm{PPV}=$ positive predictive value

S. aureus $=$ Staphylococcus aureus

SJS = Stevens-Johnson syndrome

TEN $=$ toxic epidermal necrolysis 


\section{Introduction:}

107 Epidermal necrolysis (EN) is a life-threatening condition, mainly due to drugs. According to 108 the body surface area (BSA) involved, patients are categorized as Stevens-Johnson syndrome 109 (SJS, <10\% BSA), SJS-TEN overlap (10-29\% BSA), or toxic epidermal necrolysis (TEN, or 110 Lyell syndrome, $\geq 30 \% \mathrm{BSA}) .^{1}$ Mortality ranges from 10 to $40 \%$. $^{2}$ The cornerstone of 111 treatment is supportive care. ${ }^{3}$

112 Causes of mortality in EN include specific lung involvement ${ }^{4}$ and invasive bacterial 113 infections, the most frequent and life-threatening being bloodstream infections (BSI). A 114 relationship between BSI and skin bacterial colonization was described in burns, ${ }^{5,6}$ but data 115 from EN remain scarce. Here, we aimed to investigate the epidemiology of BSI in EN and 116 assess the ability of the qualitative and quantitative results of skin cultures to predict the 117 pathogen(s) involved in BSI episodes. 


\section{Materials and methods:}

This retrospective single-center observational study included all patients admitted to the French reference center for EN between 01/2009 and 12/2017. Only patients with a final diagnosis of SJS-TEN overlap syndrome or TEN were included, as BSIs are rare in SJS. ${ }^{7}$ Diagnosis of EN was confirmed by previously published clinical criteria of EN and skin biopsy histological analysis. ${ }^{2,8}$

The following demographic, clinical, and biological data were collected from medical files: age, gender, suspected drug, SCORTEN, ${ }^{9}$ baseline and maximal detached/detachable BSA, intensive care unit admission, cyclosporin treatment, antibiotics before and during hospitalization, time to re-epithelialization, length of hospital stay, number of BSI episodes, septic shock, and hospital death.

According to the French guidelines, routine management of wound care included skin antisepsis, consisting in the application of diluted chlorhexidine (bathing or spraying) once a day, without local antibiotics, and non-sticky dressings or white petroleum jelly. Prophylactic antibiotics were not recommended. Systemic antibiotics were prescribed in case of documented invasive infection or sepsis/septic shock. ${ }^{3}$

In our routine practice, skin colonization is investigated by repeated skin cultures, performed every three days from admission to complete epithelialization, as recommended by the French guidelines. ${ }^{3}$ Skin cultures consist in applying sterile gauzes directly on the skin, on several places (usually three) chosen among the most detached/severely injured areas, and then to put those gauzes on dedicated agar plates, which are subsequently incubated at $37^{\circ} \mathrm{C}$. French guidelines also recommend daily blood cultures for all patients.

Only the first BSI episode, as defined by one (monomicrobial bacteremia) or more (polymicrobial bacteremia) pathogen(s) isolated from blood cultures, was considered for 
bacteriological analysis. Strains of the first three skin cultures collected from admission to reepithelialization for several sites of detached skin were collected: Staphylococcus aureus, Pseudomonas aeruginosa, Escherichia coli, and exogenous enterobacteria (i.e. non-E. coli enterobacteria). Three semi-quantitative classes of inoculum were defined: $<100\left(<10^{2}\right)$ colony-forming units $(\mathrm{CFU}) / \mathrm{cm}^{2}, 101-10,000\left(10^{2}-10^{4}\right) \mathrm{CFU} / \mathrm{cm}^{2}$, and $>10,000\left(>10^{4}\right)$ $\mathrm{CFU} / \mathrm{cm}^{2}$. For patients with BSI, we investigated the results of the concomitant skin culture, i.e., that performed at any time around the time of sampling the first positive blood culture, between $48 \mathrm{~h}$ before and within the $24 \mathrm{~h}$ after. We considered that skin and blood cultures were concordant when the bacteria isolated in the BSI was also isolated in the corresponding skin culture (for monomicrobial BSI), or if at least one of the bacteria of the BSI was isolated in the corresponding skin culture (for polymicrobial BSI).

Results are reported as percentages and medians (interquartiles [IQR] 25-75). The diagnostic performance of the concomitant skin culture for predicting the pathogen involved in the BSI was assessed by computing the sensitivity, the specificity, and the positive (PPV) and negative (NPV) predictive values of its qualitative results (presence or absence of the bacterium) for each pathogen involved. The odds ratio (OR), and their 95\% confidence interval, of skin cultures growing $S$. aureus, and $P$. aeruginosa for BSI episodes involving these pathogens were computed. The impact of the inoculum was also assessed by computing the OR of developing a BSI for each skin inoculum category, sterile skin cultures being the reference. Categorical data were compared using Fisher or chi-2 tests, as appropriate. Continuous data were compared using unpaired Student tests or Mann-Whitney tests, as appropriate. Two-sided $p$ values were computed and $p<0.05$ was considered significant.

The study was approved by the Institutional Review Board Mondor (IRB00011558, 2018/09/20). 
177

178

179

180

181

182

183

184

185

186

187

188

189

190

191

192

193

194

195

196

197

198

199

200

\section{Results:}

Among 166 patients referred for EN, we excluded 66 patients with SJS and two with missing data. Ninety-eight patients (55 female) were included, with a median age of 49.5 years (IQR 31.1-67.9), 40 (40.8\%) with SJS-TEN overlap syndrome and 58 (59.2\%) with TEN (Table 1). The disease was drug-induced in $82 / 98(83.7 \%)$ cases. Culprit drugs were antibiotics in 32 cases (32.6\%), antiepileptic drugs in 22 cases (22.4\%) and allopurinol in 14 cases (14.3\%)). No culprit drug was identified in 16 cases $(16.3 \%)$.

Forty-six (46.9\%) patients experienced one or more episodes of BSI during their hospital stay (total=85), after a median of seven days (IQR 0.8-13.2) following admission, resulting in an incidence of 33.3 BSI/1000 days of hospitalization/patient (Table 1). Septic shock occurred in 24/46 cases (52.2\%). Twelve patients with BSI (26\%) and nine without $(17.3 \%, \mathrm{p}=0.29)$ died. BSI occurred more frequently in patients with TEN $(n=38 / 58,65.5 \%)$ than in those with the SJS-TEN overlap syndrome $(n=8 / 40,20.0 \% ; p<0.01)$. Patients who received cyclosporin did not show a significantly higher BSI rate than those who did not.

Bacteria isolated from the first positive blood cultures were as follows (11/46 [23.9\%] BSIs were polymicrobial): S. aureus (n=17/46, 36.9\%), P. aeruginosa (n=17/46, 36.9\%), exogenous enterobacteria $(\mathrm{n}=7 / 46,15.2 \%)$, E. coli $(\mathrm{n}=1 / 46,2.2 \%)$, and others $(\mathrm{n}=15,32.6 \%)$.

The median time elapsed between hospital admission and the first BSI episode ranged from 4.5 days (0-9) for methicilline-sensitive $S$. aureus to 10 days (3-18) for $P$. aeruginosa $(\mathrm{p}=0.004)$

Ninety-six patients had one skin culture performed, 68 had two, and 34 had three or more. The three skin cultures were performed after a median of $1.0(0.0-3.9), 6.5(1.5-11.5)$, and 12.0 (6.8-14.2) days following admission. The main bacteria isolated from the first skin culture were $S$. aureus $(36 / 96,38 \%)$ and exogenous enterobacteria $(36 / 96,38 \%)$, whereas 
201

202

203

204

205

206

207

208

209

210

211

212

213

214

215

216

217

218

219

220

221

222

223

224

exogenous enterobacteria $(23 / 34,68 \%)$ and $P$. aeruginosa $(27 / 34,79 \%)$ were predominant in the third skin culture (Figure 1).

Bacteria isolated from the concomitant cutaneous samples were concordant with those responsible for BSI in 32/45 (71.1\%) cases (skin culture missing for one patient). Among those 32 skin cultures, $24(75.0 \%)$ were performed during the 48 hours preceding the BSI and $8(25.0 \%)$ during the 24 hours after. The concordance rate was 70.6\% (24/34) for monomicrobial BSI and $72.7 \%$ (8/11) for polymicrobial BSI. Among the 13 patients with discordant blood and skin culture results, $9(69.2 \%)$ had a negative skin culture and a positive blood culture, and $4(30.7 \%)$ had a positive skin culture that did not match with the positive blood culture.

The diagnostic performance of skin cultures for predicting BSI episodes was as follows: skin cultures growing S. aureus had a sensitivity of $88.2 \%$, a specificity of $60.7 \%$, a PPV of 57.7\%, and an NPV $89.4 \%$ for predicting S. aureus BSIs; for P. aeruginosa, the diagnostic performance was $75.0 \%, 58.4 \%, 50.0 \%$, and $80.9 \%$, respectively. Patients having $\geq 1$ skin culture growing S. aureus had an odds ratio (OR [95\% confidence interval]) of having a BSI episode involving the same pathogen of 17.0 [2.0-146.9] (p=0.002); for those having $\geq 1 P$. aeruginosa-positive skin culture the OR was 8 [1.9-36.4] $(\mathrm{p}=0.005)$. Post-hoc analyses of the OR of developing a BSI for increasing bacterial inocula suggested that the risk of S. aureus BSI gradually increased with the inoculum of skin culture, while that of $P$. aeruginosa did not (Figure 2).

Concerning antibiotic susceptibility at admission (skin culture 1), 8/96 patients (8.3\%) were colonized by methicillin-resistant $S$. aureus and $0 / 96(0.0 \%)$ by ceftazidime-resistant $P$. aeruginosa. Twelve days after admission (skin culture 3), these rates rose to $8 / 34$ (23.5\%, $\mathrm{p}=0.03)$ and $5 / 34(14.7 \%, \mathrm{p}<0.01)$, respectively. The 8 patients with methicillin-resistant $S$. 
aureus isolated on the third skin culture were not the same patients than the 8 who had a methicillin-resistant $S$. aureus isolated on the first skin culture.

\section{Discussion:}

In the current series, we investigated the prevalence and characteristics of BSI episodes together with the performances of skin cultures in patients with SJS-TEN overlap syndrome and TEN. Our study adds important findings on the high frequency and severity of BSI in EN with more than $10 \%$ of BSA involved, especially in TEN. Our results also highlight the importance of monitoring the skin colonization by repeated skin cultures to target the bacteria most probably involved in BSI and adapt antibiotics accordingly, even before the positivity of blood cultures. We chose to focus our analysis on the first BSI episode typically occurring at the most acute phase of the disease, while the epidermis is not healed yet, and thus is more likely to be a direct consequence of skin detachment. Indeed, in these patients, multiple BSI episodes or other severe infections such as pneumonias can occur, even after healing, especially for the most severe cases requiring intensive care unit admission and mechanical ventilation support.

We report on a high frequency of BSI in EN patients with a BSA $>10 \%$ (46.9\%) and highlighted the associated severity, illustrated by the occurrence of septic shock in nearly half of the cases. S. aureus and P. aeruginosa were the main bacteria responsible for BSI in EN, as previously reported. ${ }^{7}$ Qualitative results of skin culture showed a good concordance with BSI (71.1\%). The NPV of the concomitant skin cultures were high for $S$. aureus and $P$. aeruginosa, illustrating that patients with negative skin cultures for these pathogens were unlikely to have a BSI involving these pathogens. We also showed the importance of monitoring skin cultures over time, as we observed a switch of skin colonization from grampositive cocci at baseline to gram-negative bacilli during the hospital stay, as previously 
249 described for burn patients, ${ }^{5-7,10}$ together with a significant decrease in the susceptibility 250 patterns of the studied bacteria.

251 Our study added new data regarding the quantitative analysis of skin cultures. Indeed, skin 252 colonization with $S$. aureus or $P$. aeruginosa was associated with a higher proportion of $S$. 253 aureus and $P$. aeruginosa BSIs, respectively. Furthermore, for patients with S. aureus254 positive skin cultures, the odds ratio of having a subsequent bacteremia increased with skin 255 inoculum. Such dose-effect relationship was not observed with $P$. aeruginosa, suggesting 256 different mechanisms might be involved. For $S$. aureus, endogenous skin carriage is 257 suggested, whereas for $P$. aeruginosa, acquired skin colonization and/or digestive 258 translocation due to specific involvement of EN is possible. ${ }^{11,12}$ For other pathogens, such as 259 E. coli or other enterobacteria, digestive translocation is also suggested, as shown in patients 260 with an altered digestive barrier, including those with burns. ${ }^{13}$

261 Strengths of our study, considering the rarity of EN, were the high number of patients 262 included, the systematic process of repeated skin cultures as standard of care, and the 263 availability of complete qualitative and quantitative bacteriological analyses. The main 264 limitations include the retrospective and monocentric design and low number of BSI episodes, 265 which limit definitive conclusions to be drawn. Skin cultures were sampled in routine practice 266 on highly various anatomic sites according to the dermatologist's judgement. Furthermore, we 267 did not collect all other microbiological results, such as urine and catheter cultures. Last, it 268 can be argued that the skin cultures sampled after the first positive blood culture may be 269 affected by antimicrobial treatment initiation. However, in absence of any data, we 270 hypothesized that the cutaneous colonization was only slightly impacted by parenteral 271 antibiotics during the first 24 hours of their onset. Nevertheless, practices for skin care at the 272 acute phase are heterogeneous according to centers and a consensus is still needed for best 273 topical antiseptic or antibiotic preventive treatments of skin colonization. ${ }^{14}$ 
274 According to French guidelines and our routine practice, we do not recommend initiating 275 antibiotics on the sole basis of an isolated positive skin culture. ${ }^{3}$ However, immediate and 276 targeted antibiotics are mandatory for EN patients, as soon as invasive bacterial infection is 277 suspected (i.e., sepsis, septic shock). In that case, waiting for the complete results of BSI 278 cultures and antibiograms (that can last up to three days), the choice of the antibiotics to be 279 initiated should consider the susceptibility patterns of the bacteria carried by the patient, as 280 shown by the results of the skin culture. 


\section{References:}

300 1. Heng YK, Lee HY, Roujeau JC. Epidermal necrolysis: 60 years of errors and advances. $\mathrm{Br}$ J Dermatol. 2015 Nov;173(5):1250-4.

2. Duong TA, Valeyrie-Allanore L, Wolkenstein P, Chosidow O. Severe cutaneous adverse 303 reactions to drugs. Lancet. 2017;390(10106):1996-2011.

3. Ingen-Housz-Oro S, Duong TA, Bensaid B, et al. French National Reference Center for Toxic Bullous Dermatoses. Epidermal necrolysis French national diagnosis and care protocol (PNDS; protocole national de diagnostic et de soins). Orphanet J Rare Dis. 2018;13(1):56.

4. de Prost N, Mekontso-Dessap A, Valeyrie-Allanore L, et al. Acute respiratory failure in patients with toxic epidermal necrolysis: clinical features and factors associated with mechanical ventilation. Crit Care Med. 2014;42(1):118-28.

5. Lipový B, Holoubek J, Hanslianová M, et al. Toxic epidermal necrolysis data from the CELESTE multinational registry. Part I: Epidemiology and general microbiological characteristics. Burns. 2018;S0305-4179(18)30057-3. 
7. de Prost N, Ingen-Housz-Oro S, Duong TA, et al. Bacteremia in Stevens-Johnson syndrome and toxic epidermal necrolysis: epidemiology, risk factors, and predictive value of skin cultures. Medicine. 2010;89(1):28-36.

8. Valeyrie-Allanore L, Bastuji-Garin S, Guégan S, et al. Prognostic value of histologic features of toxic epidermal necrolysis. J Am Acad Dermatol. 2013;68(2):29-35.

9. Bastuji-Garin S, Fouchard N, Bertocchi M, Roujeau JC, Revuz J, Wolkenstein P. SCORTEN: a severity-of-illness score for toxic epidermal necrolysis. J Invest Dermatol. 2000;115(2):149-53.

10. Nasser S, Mabrouk A, Maher A. Colonization of burn wounds in Ain Shams University Burn Unit. Burns. 2003;29(3):229-33.

11. Gendreau S, Amiot A, Le Baleur $\mathrm{Y}$, et al. Gastro-intestinal involvement in StevensJohnson syndrome and toxic epidermal necrolysis: a retrospective case series. Br J Dermatol. 2018 Nov 15. doi: 10.1111/bjd.17428.

12. Fava $P$, Astrua $C$, Cavaliere $G$, et al. Intestinal involvement in toxic epidermal necrolysis. A case report and review of literature. J Eur Acad Dermatol Venereol. 2015;29(9):1843-5.

13. Earley ZM, Akhtar S, Green SJ, et al. Burn Injury Alters the Intestinal Microbiome and Increases Gut Permeability and Bacterial Translocation. PLoS One. 2015;10(7):0129996.

14. Castillo B, Vera N, Ortega-Loayza AG, et al. Wound care for Stevens-Johnson syndrome and toxic epidermal necrolysis. J Am Acad Dermatol. 2018;79(4):764-767.

(1)




\section{Legends of Figures:}

\section{Figure 1:}

346 Percentage of skin cultures positive for the bacterial species of clinical interest.

347 Percentages were compared for each bacterial species on skin cultures 1, 2 and 3 using Chi2 test. Differences with $\mathrm{p}<0.05$ were considered significant.

349

\section{Figure 2:}

351 Odds ratio of developing S. aureus and P. aeruginosa bloodstream infections (BSI) according

352 to the inoculum of skin culture, expressed in colony-forming units $(\mathrm{CFU}) / \mathrm{cm}^{2}$.

353 For S. aureus, but not for $P$. aeruginosa, odds ratios of BSIs increased with skin inoculum.

354

355

356

357

358

359 
366 Table 1: Characteristics and comparison of patients with and without a bloodstream infection.

367

\begin{tabular}{|c|c|c|c|c|}
\hline Patient characteristics & $\begin{array}{l}\text { All patients } \\
\mathrm{n}=98\end{array}$ & $\begin{array}{l}\text { Patients with } \\
\text { bloodstream infection } \\
\mathrm{n}=46\end{array}$ & $\begin{array}{l}\text { Patients without } \\
\text { bloodstream infection } \\
\mathrm{n}=52\end{array}$ & P-value \\
\hline Female, $\mathrm{n}(\%)$ & $55(56)$ & $21(45.6)$ & $34(65.4)$ & 0.049 \\
\hline Median age, years (range) & $49.5(15-99)$ & $54.5(15-99)$ & $47.5(18-83)$ & 0.23 \\
\hline $\begin{array}{l}\text { Median SCORTEN at baseline } \\
\text { (range) }\end{array}$ & $2(0-5)$ & $2(1-5)$ & $2(0-5)$ & 0.001 \\
\hline $\begin{array}{l}\text { Median BSA involved at baseline, } \\
\% \text { (range) }\end{array}$ & $15(0-95)$ & $20(0-80)$ & $10(0-95)$ & 0.008 \\
\hline $\begin{array}{l}\text { Median maximal BSA involved, \% } \\
\text { (range) }\end{array}$ & $30(10-100)$ & $60(10-100)$ & $25(10-100)$ & $<0.0001$ \\
\hline $\begin{array}{l}\text { Median time to cutaneous re- } \\
\text { epithelialization, days (range) }\end{array}$ & $5(0-19)$ & $7(2-19)$ & $5(0-11)$ & 0.07 \\
\hline Treatment with cyclosporin, n (\%) & $57(58.1)$ & $26(56.5)$ & $31(67.4)$ & 0.75 \\
\hline $\begin{array}{l}\text { Median length of hospitalization, } \\
\text { days (range) }\end{array}$ & $20(4-116)$ & $28.5(4-116)$ & $15(4-43)$ & 0.0001 \\
\hline $\begin{array}{l}\text { Antibiotics before admission, } \mathrm{n} \\
(\%)\end{array}$ & $67(68.4)$ & $30(65.2)$ & $37(71.2)$ & 0.53 \\
\hline $\begin{array}{l}\text { Antibiotics during hospitalization, } \\
\mathrm{n}(\%)\end{array}$ & $81(82.7)$ & $45(97.8)$ & $36(69.2)$ & 0.0001 \\
\hline $\begin{array}{l}\text { Intensive care unit admission, } \mathrm{n} \\
(\%)\end{array}$ & $49(50)$ & $34(73.9)$ & $15(28.8)$ & $<0.0001$ \\
\hline Hospital death, n (\%) & $21(20.4)$ & $12(26)$ & $9(17.3)$ & 0.29 \\
\hline
\end{tabular}

368 BSA: body surface area 
Figure 1:

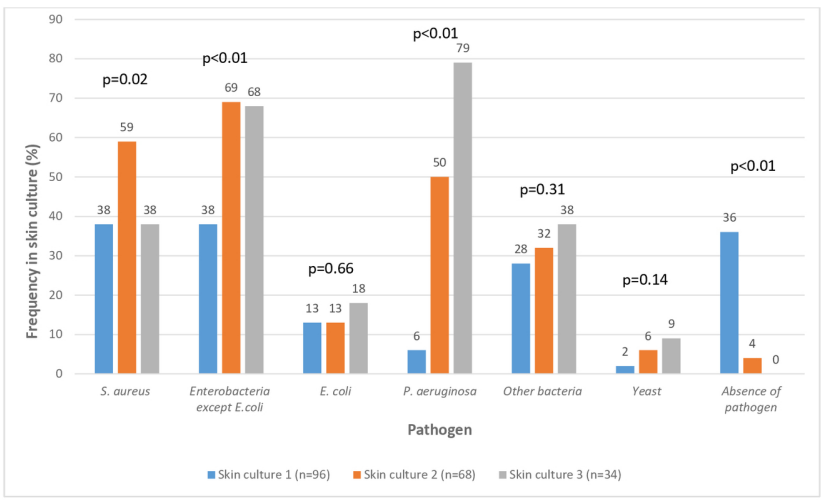


Staphylococcus aureus

Pseudomonas aeruginosa

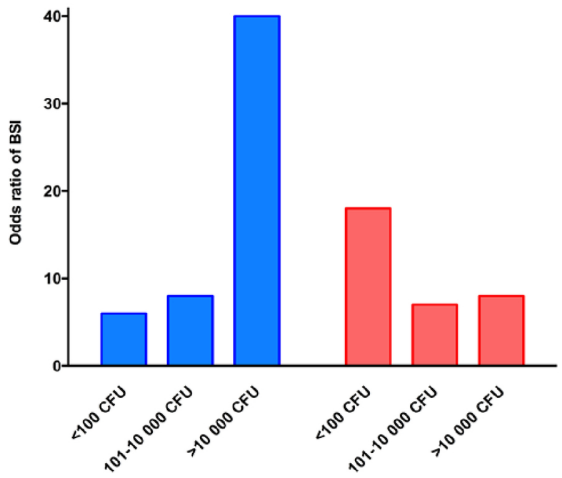

